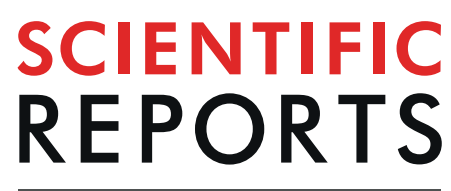

natureresearch

\title{
Impact of multisection and immunohistochemistry in lymph node staging of Gastric Carcinoma - Case series
}

\author{
Fernando A. V. Santos ${ }^{1,2^{*}}$, Ana P. Drummond-Lage $\mathbb{1}^{1}{ }^{1}$, Alberto J. A. Wainstein $\mathbb{1}^{1}$, \\ Marco A. Dias-Filho², Paulo R. Savassi-Rocha ${ }^{2}$ \& Tulio P. Navarro
}

Gastric carcinoma (GC) locoregional recurrence may occur even in cases where the tumor has been completely resected, possibly due to lymph node (LN) micrometastases. It is estimated that in $10 \%$ to $30 \%$ of cases, LN micrometastases are not detected by a conventional method for histological assessment of $L N$ metastases with hematoxylin-eosin (HE). A cross-sectional study assessed 51 patients with GC by histological evaluation of the $L N$ micrometastases through $L N$ multi sectioning associated with immunohistochemistry analysis with monoclonal antibodies AE1 and AE3. Total gastrectomy was performed in $51 \%$ of patients. The total number of resected $L N$ nodes was 1698 , with a mean number of resected $L N$ of $33.3 \pm 13.2$ per surgical specimen, of which 187 had metastasis. After the application of LN multisection and immunohistochemistry, $\mathrm{LN}$ micrometastases were found in $45.1 \%$ of the cases. LN staging changed in $29.4 \%$, and tumor staging changed in $23.5 \%$ of the cases. In patients initially staged as pNO, LN staging and tumor staging changed, both in $19.2 \%$ of the cases. In patients initially staged as pN1 or more, $\mathrm{LN}$ staging changed in $40.0 \%$ of them, and tumor staging changed in $28.0 \%$ of the cases. The accuracy of $\mathrm{HE}$ for the histological staging of $L N$ tumoral involvement was $76 \%$, which was considered insufficient for CG patients staging. Investigation of $L N$ micrometastasis through $L N$ multisection and immunohistochemistry should be performed, particularly in cases where the presence of blood and lymphatic vessel invasion has been identified after conventional histological analysis, as well as in patients with advanced GC.

Gastric carcinoma (GC), despite the recent decrease observed in its incidence, remains the second most common cause of cancer death in the world, with more than 600,000 deaths per year. The main chance of cure of this neoplasm is on surgical resection. The standard procedure for the treatment of this condition is radical gastrectomy, which includes gastric resection with surgical margins free of neoplasia, associated with extended locoregional lymphadenectomy ${ }^{1-3}$.

Accurate tumor staging is one of the leading factors in the definition of the therapeutic strategy. From the exact knowledge of the extent of tumor dissemination, it is possible to define, with greater security, the best therapeutic approach for each patient, and, consequently, avoid incomplete or excessive treatments $\mathrm{s}^{4,5}$.

Recent literature has discussed the role of lymph node (LN) micrometastasis in GC. Conceptually, micrometastases are metastases of sizes between $0.2 \mathrm{~mm}$ and $2.0 \mathrm{~mm}$ (5). Its incidence varies between $10 \%$ to $30 \%{ }^{6,7}$. Currently, many authors admit that its presence is associated with a worse prognosis and that the clinical behavior of these patients is like those with lymph node involvement by metastasis ${ }^{8}$. In the submucosal GC (T1b), with the absence of LN metastases and micrometastases, the five-year survival rate is close to $100 \%$, being significantly higher than in micrometastases positive cases $(82 \%)^{6}$.

The presence of micrometastasis may be related to locoregional recurrence, as one can see in wholly resected GC initially staged without LN metastasis at traditional histological analysis using hematoxylin-eosin (HE). It is estimated that between $22 \%$ to $66 \%$ of patients with tumor stages I and II, submitted to radical surgical treatment, including individuals without LN metastasis, died due to local or distance tumor recurrence ${ }^{6,9}$.

${ }^{1}$ Faculdade Ciencias Medicas de Minas Gerais, Alameda Ezequiel Dias 275, Belo Horizonte, MG, 30130-110, Brazil. 'Universidade Federal de Minas Gerais, Alfredo Balena 189, Belo Horizonte, 30130-100, Brazil. *email: fernandoavsantos@gmail.com 


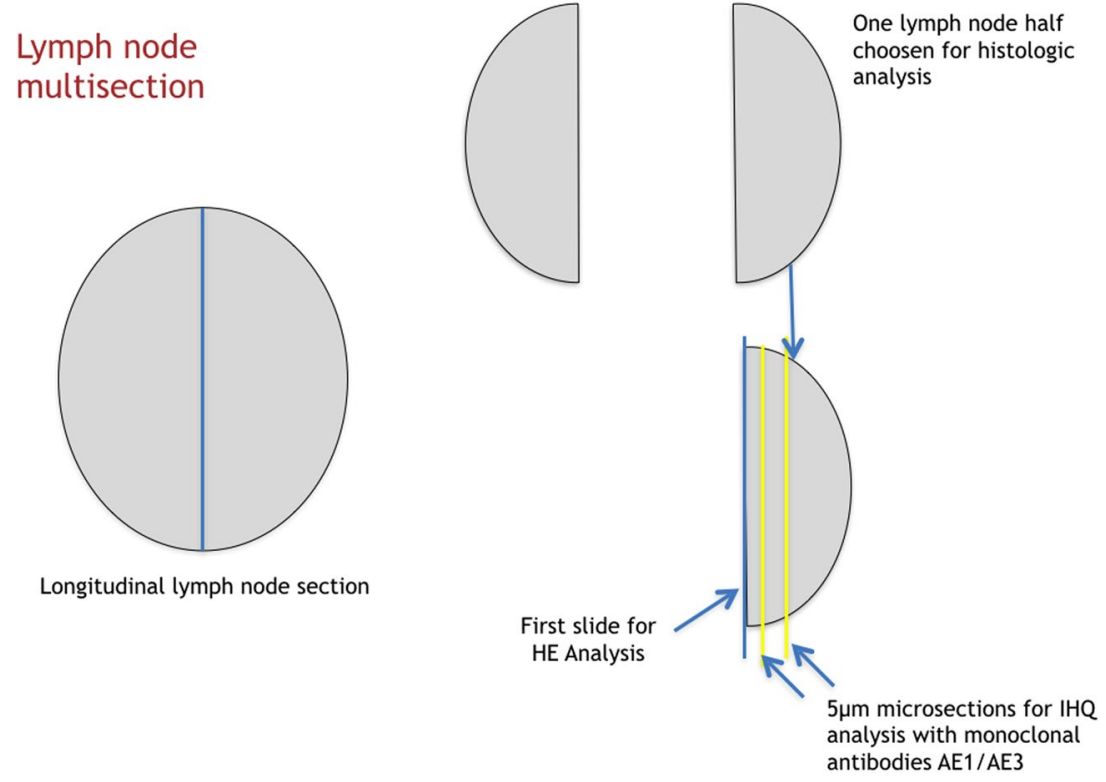

Figure 1. The multisection technique for histological and immunohistochemical analysis for lymph node staging.

The routine evaluation of LN resected during radical gastrectomy, which includes analysis of only one LN histological section with $\mathrm{HE}$, can result in the non-identification of micrometastases. This may generate a suboptimal LN staging and, therefore, lead to therapeutic measures that may be insufficient for the accuracy of the tumor staging ${ }^{10,11}$.

Thus, to reduce the chances of GC understaging, this research has the goal to assess the presence and frequency of LN micrometastasis, analyze the accuracy between conventional histological assessment by HE and immunohistochemical (IHC) evaluation with monoclonal antibodies AE1 and AE3associated with LN multisection, and to identify subgroups at higher risk of LN micrometastases.

\section{Materials and Methods}

Patients. We analyzed GC patients submitted to radical gastrectomy, associated with D2 lymphadenectomy, according to the Japanese Gastric Cancer Association guidelines (JGCA) ${ }^{4}$. Experienced and trained oncologic surgeons performed all surgeries.

Exclusion criteria were related to patients who had previous gastric surgery and had been submitted to neoadjuvant treatment.

Lymph node evaluation. One pathologist examined tumor specimens. The LN involvement by metastasis and micrometastases in perigastric and extra perigastric LN nodes were analyzed. Each end every LN resected without metastases, identified by HE histological analysis, regardless of the initial LN staging, had a longitudinal cut in its structure, and one of its halves were submitted to three microsections, with an interval of $5 \mu \mathrm{m}$ among slices (Fig. 1). The first one for HE (Fig. 2A), and the other two for IHC analysis (Fig. 2B). Tissue sections from paraffin blocks were deparaffinized with xylene and rehydrated with graded ethanol dewaxed. Endogenous peroxidase was blocked with $\mathrm{H}_{2} \mathrm{O}_{2}(3 \%)$ for $30 \mathrm{~min}$. Afterward, the section was incubated with primary antibody AE1/AE3 (Roche/Ventana), overnight at $4{ }^{\circ} \mathrm{C}$. The secondary antibody used was biotinylated goat anti-polyvalent followed by streptavidin-biotin peroxidase-conjugated enzyme. The substrate and 3,3' diaminobenzidine and section were incubated for $10 \mathrm{~min}$ at room temperature in the dark. The positivity of the IHQ reaction was confirmed when desmoplastic cells were identified into the LN stroma by their dark brown color.

Definition of lymph node-positive micrometastases. Micrometastases were defined as referring to tumor cell clusters between $0.2 \mathrm{~mm}$ and $2.0 \mathrm{~mm}$ in the greatest dimension. Isolated tumors cells, which are cells or cell clusters measuring less than $0.2 \mathrm{~mm}$ in the greatest dimension, were not considered for statistical analysis. The GC tumor staging followed the pre-established standards from the 8th AJCC-TNM system ${ }^{5}$.

Statistical analysis. For the sample calculation, considering a margin of error of $5 \%, 95 \%$ confidence intervals, and $10 \%$ prevalence of the proportion of micrometastases in GC, a sample of 47 individuals were found. The descriptive analysis of qualitative variables was performed by absolute and relative frequencies, for quantitative variables, by the mean and standard deviation. The comparison of means between groups was performed using the Wilcoxon non-parametric test. The association between categorical variables were evaluated by Fisher's exact test or the chi-square test, when appropriate. 

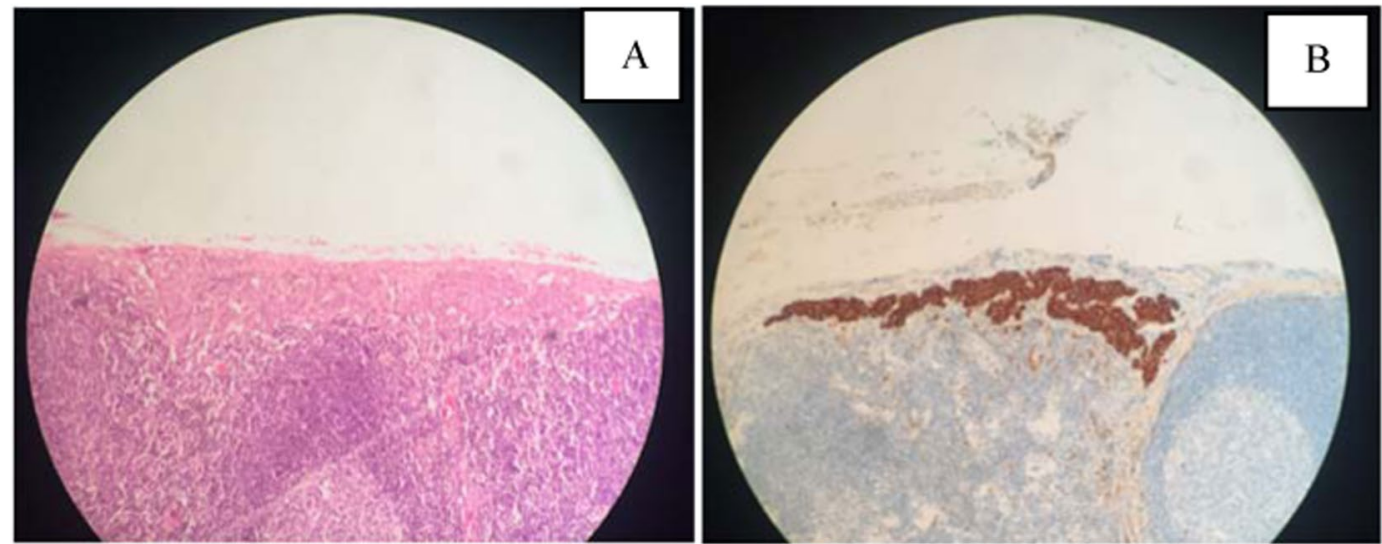

Figure 2. (A) Microscopic image of lymph node micrometastasis by Hematoxylin/Eosin. (B) Microscopic image of lymph node micrometastatis by Immunohistochemistry(AE1/AE3).

\begin{tabular}{|l|l|l|l|}
\hline $\begin{array}{l}\text { Tumoral staging before MS/IHC } \\
\text { evaluation - number of cases }\end{array}$ & $\begin{array}{l}\text { Initial tumoral } \\
\text { staging }\end{array}$ & $\begin{array}{l}\text { Tumoral staging after MS/IHC } \\
\text { evaluation - number of cases }\end{array}$ & $\begin{array}{l}\text { Final tumoral } \\
\text { staging }\end{array}$ \\
\hline 1 & IA & 0 & IA \\
\hline 1 & IB & 1 & IB \\
\hline 1 & IIA & 1 & IIA \\
\hline 2 & IIB & 1 & IIB \\
\hline 0 & IIIA & 2 & IIIA \\
\hline
\end{tabular}

Table 1. Staging and tumor restaging before and after the detection of micrometastases by IHC and MS in patients operated for gastric cancer without lymph node metastases by hematoxylin-eosin $(n=5)$. MS - Lymph node multisection, IHC - immunohistochemistry.

\section{Results}

The sample was composed of 51 patients, $51 \%$ females. The mean age was $62.7 \pm 14.8$ years. Total gastrectomy was performed in $51 \%$ of the patients. The average size of the tumors was $4.6 \mathrm{~cm} \pm 2.4 \mathrm{~cm}$, and predominantly in the distal location (64.7\%). The total number of LN resected was 1698 , with an average number of $33.3 \pm 13.2$ per surgical piece, of which 187 had metastasis. The average percentage of metastatic LNs was $11.0 \%$. The tumor depth was serosa $(35.3 \%)$, subserosa (11.8\%), muscularis propria $(21.5 \%)$, submucosa $(15.7 \%)$, and mucosa $(15.7 \%)$. The diffuse type, according to Laurén's classification, was found in $41.2 \%$ of cases, followed by the intestinal type $(37.2 \%)$ and the mixed type (21.6\%). Blood vessels, lymphatic, and neural invasion occurred in $47.1 \%, 62.8 \%$, and $35.3 \%$ of the cases, respectively.

Regarding the LN assessment by HE, in 26 cases, LN was not identified (pN0), and 25 cases had LN involvement identified on a histological assessment by $\mathrm{HE}$, changing $\mathrm{LN}$ staging from $\mathrm{pN} 1$ to $\mathrm{pN} 3 \mathrm{~B}$.

After the histological evaluation of LN with the application of IHC and LNMS, LN micrometastases were found in 23 cases (45.1\%). Five cases were initially staged as pNO by HE, and 18 cases belonged to the group of patients with known LN metastasis.

The LN staging by HE detection showed an accuracy of $76.0 \%$, sensitivity of $78.0 \%$, and specificity of $75.0 \%$. In its turn, the positive and negative predictive values of histological assessment by HE in the detection of any LN metastasis were $72.0 \%$ and $80.0 \%$, respectively.

IHC and LN MS diagnosed LN micrometastases in five cases (19.2\%) out of the subgroup of $26 \mathrm{pN} 0$ patients. The nodal staging changed in all these five cases (19.2\%), being pN1 in four cases and pN2 in one case (Table 1).

As to the subgroup of 25 patients with LN metastases diagnosed by HE, in 18 (72.0\%) of them, micrometastases were also identified by IHC, and LN MS. Ten of these patients (40.0\%) underwent a restaging of the LN involvement. In seven of them (28.0\%), the tumor staging also suffered changes after evaluation with IHC and LN MS (Tables 2 and 3).

Among the 51 patients evaluated, LN staging has changed in 15 of them (29.4\%) after LN assessment by IHC and MS. The final tumor restaging occurred in 12 cases $(23.5 \%)$, i.e., in $80 \%$ of the cases in which the LN staging changed, there was also a change in the final tumoral staging (Table 4 ).

Blood and lymphatic vessel invasion at $\mathrm{HE}$ analysis were significantly associated with the presence of micrometastases, at an incidence of $65 \%(p=0,026)$ and $87 \%(p=0,001)$, respectively. Advanced gastric cancer was considered a risk factor for LN micrometastases in comparison with early gastric cancer (Table 5). 


\begin{tabular}{|l|l|l|l|}
\hline $\begin{array}{l}\text { Lymph node staging before } \\
\text { MS/IHC- number of cases }\end{array}$ & $\begin{array}{l}\text { Initial Lymph } \\
\text { node staging }\end{array}$ & $\begin{array}{l}\text { Lymph node staging after } \\
\text { MS/IHQ - number of cases }\end{array}$ & $\begin{array}{l}\text { Final Lymph } \\
\text { node staging }\end{array}$ \\
\hline 7 & pN1 & 0 & pN1 \\
\hline 1 & pN2 & 7 & pN2 \\
\hline 2 & pN3a & 0 & pN3a \\
\hline 0 & pN3b & 3 & pN3b \\
\hline
\end{tabular}

Table 2. Staging and restaging of lymph node involvement by micrometastases, after evaluation by IHC and MS in gastric cancer patients operated with known lymph node metastases by hematoxylin-eosin $(n=10)$. MS Lymph node multisection, IHC - immunohistochemistry.

\begin{tabular}{|l|l|l|l|}
\hline $\begin{array}{l}\text { Tumoral staging before MS/IHC } \\
\text { evaluation: number of cases }\end{array}$ & $\begin{array}{l}\text { Initial tumoral } \\
\text { staging }\end{array}$ & $\begin{array}{l}\text { Tumoral staging after MS/IHC } \\
\text { evaluation: number of cases }\end{array}$ & $\begin{array}{l}\text { Final tumoral } \\
\text { staging }\end{array}$ \\
\hline 2 & IIA & 0 & IIA \\
\hline 2 & IIB & 2 & IIB \\
\hline 2 & IIIA & 2 & IIIA \\
\hline 1 & IIIB & 1 & IIIB \\
\hline 0 & IIIC & 2 & IIIC \\
\hline
\end{tabular}

Table 3. Tumoral staging and restaging before and after the detection of micrometastasis to IHC and MS in patients operated by gastric carcinoma with known lymph node metastasis to hematoxylin-eosin $(\mathrm{N}=7)$. MS Lymph node multisection, IHC - immunohistochemistry.

\begin{tabular}{|l|l|l|l|l|}
\hline Patient \# & $\begin{array}{l}\text { Lymph node staging } \\
\text { before MS/IHC }\end{array}$ & $\begin{array}{l}\text { Tumoral staging } \\
\text { before MS/IHC }\end{array}$ & $\begin{array}{l}\text { Lymph node staging } \\
\text { after MS/IHC }\end{array}$ & $\begin{array}{l}\text { Tumoral staging } \\
\text { after MS/IHC }\end{array}$ \\
\hline 2 & N3a & IIIA & N3b* & IIIB** \\
\hline 4 & N3a & IIIB & N3b* & III *** \\
\hline 11 & N1 & IIB & N2* & IIIA** \\
\hline 16 & N1 & IIB & N2* & IIIA* \\
\hline 18 & N1 & IIIA & N2* & IIIA \\
\hline 20 & N1 & IIIA & N2* & IIIA \\
\hline 25 & N1 & IIIA & N2* & IIIA \\
\hline 29 & N0 & IA & N1* & IB** \\
\hline 34 & N1 & IIA & N2* & IIB** \\
\hline 35 & N0 & IB & N1* & IIA** \\
\hline 36 & N1 & IIA & N2* & IIB** \\
\hline 39 & N0 & IIB & N1* & IIIA** \\
\hline 41 & N2 & IIIA & N3b* & IIIC** \\
\hline 45 & N0 & IIA & N1* & IIIA** \\
\hline 50 & N0 & & IIB** \\
\hline
\end{tabular}

Table 4. Lymph node staging and initial and final tumor staging in the subgroup of patients reestablished after identification of lymph node micrometastases by IHC and MS techniques in gastric carcinoma $(\mathrm{n}=15)$. *Cases where there was a change in the category of lymph node staging, ${ }^{*}$ Cases in which tumor staging was changed, MS - lymph node multisection, IHC - immunohistochemistry.

\section{Discussion}

In the Middle West, GC is usually diagnosed at an advanced stage and with LN metastases. Those metastases are an independent factor for poor prognosis, reducing patient disease-free survival and overall survival. The detection of LN metastases is critical for the correct classification of tumoral staging and should be investigated, to allow the best prognostic definition of CG, and to provide the means for the stratification of individuals who could benefit or not from adjuvant chemotherapy ${ }^{1,5,12}$.

The frequency of micrometastases in GC is estimated between $10 \%$ to $30 \%$. It may vary not only by factors directly related to the tumor itself but also by the method used for their identification. It is believed that LNmicrometastases may be responsible for tumor recurrence in patients whose tumors were resected entirely, even in cases in which there were no LN metastases ${ }^{13,14}$.

The latest edition of the AJCC/TNM classification for GC establishes that micrometastases when found, must be notified by the acronym pNmi. However, guidelines on how and when to carry out their research were not defined, and its presence should not affect tumor and LN node GC staging 5 . 


\begin{tabular}{|c|c|c|c|}
\hline \multirow[b]{2}{*}{ Variables } & \multicolumn{2}{|c|}{ Micrometastases } & \multirow[b]{2}{*}{ p-valor } \\
\hline & No n(\%) & Yes n (\%) & \\
\hline Gender & & & 0.264 \\
\hline Female & $12(42.9)$ & $14(60.9)$ & \\
\hline Male & $16(57.1)$ & $9(39.1)$ & \\
\hline Age (years) & & & 1.000 \\
\hline Mean \pm standard deviation & $62.8 \pm 15.0$ & $62,7 \pm 14.8$ & \\
\hline Gastrectomy & & & 0.577 \\
\hline Subtotal & $15(53.6)$ & $10(43.5)$ & \\
\hline Total & $13(46.4)$ & $13(56.5)$ & \\
\hline Tumor size (cm) & & & 0.239 \\
\hline Mean \pm standard deviation & $4.3 \pm 2.6$ & $5.0 \pm 2.2$ & \\
\hline Stomach site & & & 0.769 \\
\hline Body/Proximal & $9(32.1)$ & $9(39.1)$ & \\
\hline Distal & $19(67.9)$ & $14(60.9)$ & \\
\hline Serous tumor depth & & & 0.141 \\
\hline No & $21(75.0)$ & $12(52.2)$ & \\
\hline Yes & $7(25.0)$ & $11(47.8)$ & \\
\hline Laurén & & & 0.225 \\
\hline Diffuse & $11(39.3)$ & $10(43.5)$ & \\
\hline Intestinal & $13(46.4)$ & $6(26.1)$ & \\
\hline Mixed & $4(14.3)$ & $7(30.4)$ & \\
\hline \multicolumn{4}{|c|}{ Vascular/Lymphatic/Neural invasion } \\
\hline Vascular & $9(32.1)$ & $15(65.2)$ & 0.026 \\
\hline Lymphatic & $12(42.9)$ & $20(87.0)$ & 0.001 \\
\hline Neural & $7(25.0)$ & $11(47.8)$ & 0.141 \\
\hline
\end{tabular}

Table 5. Comparison of the different variables in patients submitted to radical gastrectomy about the presence of micrometastases in gastric carcinoma $(n=51)$. p-test significant when $<0.05$. It was shown that $60 \%$ of the advanced gastric cancer patients had micrometastases compared to $12,5 \%$ of the early gastric cancer group $(\mathrm{p}=0,002)$.

The best method for the histological diagnosis of LN micrometastases is not known, and AJCC does not provide any guidance on this subject. In the literature, there is no definition of how many LN MS must be done. Some authors propose just one cut, and others suggest the whole LN sectioning. This can impair the implementation of LN micrometastases search at pathology laboratories due to the work overload caused by this procedure ${ }^{15-18}$. In either way, when searching for LN micrometastases, it is recommended IHC using anti-cytokeratin antibodies as it increases the micrometastases detection rate ${ }^{19,20}$.

In a study published by Isosaki et al., the $\mathrm{LN}$, resected from 111 patients submitted to radical gastrectomy were analyzed for the presence of metastases and micrometastases based on HE analysis and full sectioning of the LN, resulting in the assessment of 58,430 slides The authors concluded that, when comparing the diagnostic efficacy of LN metastases based on one section, three sections, and the entire LN sectioning, the three-sectioning method was the most cost-effective of all ${ }^{21}$. This finding was also reported by other authors, even in tumors of different primary sites ${ }^{15,22-24}$.

In the present study, three LN sections were performed. It added 59 metastatic LN to the initially 187 metastatic LN found, and the incidence of metastatic LN rose from $11.0 \%$ to $14.5 \%$ (246 of 1698 lymph nodes). The global frequency of micrometastases was $45.1 \%$. The accuracy of histological assessment using HE was $76 \%$. The importance of $\mathrm{LN}$ multisection is highlighted by the fact that the micrometastases and, sometimes, the LN metastases, could only be identified in additional cuts from the LN MS. In two cases (3.9\%), they were found exclusively in the third section level of the LN, and, in these cases, the LN staging changed from pN0 to pN1, and the tumor staging went from IB to IIA. Thus, if the MS had not been performed, the LN micrometastases would have never been found, and it would have been a cause of tumor downstaging.

Other studies assessing the presence of micrometastases, not only in GC but also in colonic, esophageal, head and neck, and gynecological tumors, also observed the same findings. Such studies attest that, regardless of the tumor staging, the conventional histological evaluation, which is based on just one cut in the LN structure, does not stage the metastatic LN involvement adequately ${ }^{13,15,16,25}$.

It is not clear for which group of patients the micrometastases research would be indicated. It is possible that the finding of specific pathological variables at the HE analysis could serve as a guide for the regular survey of micrometastases in LN resected during gastrectomy.

Considering the variables analyzed in this study (age, sex, gastrectomy type, tumor size, histological type, depth of involvement of gastric wall, tumor location, number of lymph nodes resected, lymphatic vessel invasion, blood vessel invasion, and neural invasion), only the blood and lymphatic vessel invasion showed statistical significance concerning the presence of micrometastases. 
Nevertheless, when considering two different groups, one with initial and other with advanced GC, it was noted that the incidence of micrometastatic LN was significantly higher in the second group. These observations were also reported by other authors ${ }^{14,22,26,27}$. In the overall analysis of the results obtained in the present study, it should be noted that, with the addition of LN MS and IHC, the final LN and tumor staging changed significantly. Out of the 51 patients studied, one-third of them had their LN staging changed, and $23.5 \%$ of them had a reclassification of their final tumor staging. In $80 \%$ of the cases in which there was a change in the final LN staging, the final tumor staging changed as well.

The non-identification of LN micrometastases may interfere negatively in the analysis result of the surgical treatment of GC. It may be a cause of misunderstanding of patients' survival rates. For instance, patients with a lower category of LN involvement could have worse survival rates compared with others in a more advanced cancer stage, probably because of the non-identification of micrometastases by $\mathrm{HE}$ analysis ${ }^{28-30}$.

Lee et al., in 2015, prospectively evaluated the prognostic value of micrometastases and whether these should be considered or not in the pathological LN staging of GC. Analyzing 482 patients undergoing a curative gastrectomy and examining all the resected and negative LN for metastases by HE, even in those cases in which the initial LN staging was $\mathrm{pN} 1$ or greater, there was a change of pathological LN staging in $15.6 \%$ of the cases. When LN micrometastases were considered in the pathological LN staging, there was a higher accuracy of results: most patients initially considered as $\mathrm{pN} 2$ were, in fact, $\mathrm{pN} 3 \mathrm{a}$. With this change, the survival curve was within the normal range and maintained throughout the years. The authors urged the need for the inclusion of micrometastases in the LN node staging system ${ }^{31}$.

In the present study, after the completion of the LN MS and IHC analysis, LN micrometastases were found in five of the 26 cases initially staged as pN0. The LN and final tumor staging have changed in all these cases. In four situations, the final LN staging increased to N1, and one situation increased to N2. This is extremely important and demonstrates the fragility of LN staging when using only HE.

So far, adjuvant chemotherapy is not indicated for patients whose LN micrometastases were detected. According to guidelines from the National Comprehensive Cancer Network (NCCN), as well as from the JGCA, adjuvant chemotherapy has only been proved to be beneficial in patients with advanced GC, and in patients with LN metastases, increasing both disease-free survival and overall survival ${ }^{32-34}$.

The study has limitations. As its primary focus regards the histological and immunohistochemical diagnosis of lymph node metastasis, there is no data related to clinical outcomes, including survival and recurrence.

\section{Conclusions}

The application of lymph node multisection and IHC to assess lymph node staging in GC patients submitted to radical gastrectomy demonstrate that the accuracy of the conventional HE method for the histological staging of lymph node involvement is insufficient. Also, the research of micrometastases through lymph nodes multisection and IHC should be undertaken, particularly in cases in which there was the identification of the presence of blood and lymphatic vessel invasion, as well as in patients with advanced CG.

\section{Compliance with Ethical Standards.}

- All procedures performed in this study were per the ethical standards of the institutional and/or national research committee and with the 1964 Helsinki declaration and its later amendments or comparable ethical standards.

- The Universidade Federal de Minas Gerais Ethics Committee previously approved this project.

- Informed consent was obtained from all individual participants included in the study.

Received: 23 May 2019; Accepted: 23 January 2020;

Published online: 24 February 2020

\section{References}

1. Zilberstein, B. et al. Brazilian consensus in gastric cancer: guidelines for gastric cancer in Brazil. Arq Bras Cir Dig. 26, 2-6 (2013).

2. Dubecz, A. et al. Does the incidence of adenocarcinoma of the esophagus and gastric cardia continue to rise in the twenty-first century? A SEER database analysis. J Gastrointest Surg. 18, 124-9 (2014).

3. Karimi, P., Islami, F., Anandasabapathy, S., Freedman, N. D. \& Kamangar, F. Gastric Cancer: Descriptive Epidemiology, risk factors, screening, and prevention. Cancer Epidemiol Biomarkers Prev. 23, 700-13 (2014).

4. Japanese Gastric Cancer Association. Japanese gastric cancer treatment guidelines 2014 (ver. 4). Gastric Cancer. 20, 1-19 (2017).

5. Amim, M. B. et al. The Eighth Edition AJCC Cancer Staging Manual: Continuing to build a bridge from a population-based to a more "personalized" approach to cancer staging. Cancer J Clin. 67, 203-220 (2017).

6. Jeuck, T. L. A. \& Wittekind, C. Gastric carcinoma: stage migration by immunohistochemically detected lymph node micrometastases. Gastric Cancer. 18, 100-8 (2015)

7. Nakajo, A. et al. Detection and prediction of micrometastasis in the lymph nodes of patients with pN0 gastric cancer. Ann Surg Oncol. 8, 158-62 (2001).

8. Yasuda, K. et al. Prognostic effect of lymph node micrometastasis in patients with histologically node-negative gastric cancer. Ann Surg Oncol. 9, 771-4 (2002).

9. Scheunemann, P. et al. Occult disseminated tumor cells in lymph nodes of patients with gastric carcinoma. A critical appraisal of assessment and relevance. Langenbecks Arch Surg. 394, 105-13 (2009).

10. Saito, H. et al. Recurrence in early gastric cancer- the presence of micrometastasis in lymph node of node-negative early gastric cancer patient with recurrence. Hepatogastroenterology. 54, 620-4 (2007).

11. Natsugoe, S. et al. Lymph node micrometastasis in gastrointestinal tract cancer - A clinical aspect. Int J Clin Oncol. 18, 752-61 (2013).

12. He, X. et al. Validation of the American Joint Committee on Cancer (AJCC) 8th edition stage system for gastric cancer patients: a population-based analysis. Gastric Cancer. 21, 1-10 (2018). 
13. Pereira, M. A. et al. Detection of occult lymph node tumor cells in node-negative gastric cancer patients. Arq Bras Cir Dig. 30, 30-4 (2017).

14. Lee, C. M., Park, S. S. \& Kim, J. H. Current status and scope of lymph node micrometastasis in gastric cancer. J Gastric Cancer. 15, $1-9(2015)$.

15. Wong, Y.-P. et al. Comparative analysis between multilevel sectioning with conventional haematoxylin and eosin staining and immunohistochemistry for detecting nodal micrometastases with stage I and II colorectal cancers. Asian Pac J Cancer Prev. 15, $1725-30$ (2014).

16. Ensani, F. et al. Improved detection of metastases by step sectioning and immunohistochemical staining of axillary sentinel nodes in patients with breast carcinoma. Asian Pac J Cancer Prev. 14, 5731-4 (2013).

17. Hata, M. et al. Entire-volume serial histological examination for detection of micrometastases in lymph nodes of colorectal cancers. Pathol Oncol Res. 17, 835-41 (2011).

18. Yared, M. A. et al. Recommendations for sentinel lymph node processing in breast cancer. Am J Surg Pathol. 26, 377-82 (2002).

19. Guo, C.-B., Li, Y.-A. \& Gao, Y. Immunohistochemical staining with cytokeratin combining semi-serial sections for detection of cervical lymph node metastases of oral squamous cell carcinoma. Auris Nasus Larynx. 34, 347-51 (2007).

20. Zhou, Y., Zhang, G.-J., Wang, J., Zheng, K.-Y. \& Fu, W. Current status of lymph node micrometastasis in gastric cancer. Oncotarget. 8, 51963-51969 (2017)

21. Isozaki, H. \& Okajima, K. F. K. Histological Evaluation of Lymph Node metastasis on Serial Sectioning in Gastric Cancer with radical lymphadenectomy. Hepatogastroenterology. 44, 1133-6 (1997).

22. Lee, E. et al. Prognostic relevance of immunohistochemically detected lymph node micrometastasis in patients with gastric carcinoma. Cancer. 94, 2867-73 (2002).

23. Kim, C. H. et al. Pathologic Ultrastaging Improves Micrometastasis Detection in Sentinel Lymph Nodes During Endometrial Cancer Staging. Int J Gynecol Cancer. 23, 964-970 (2013).

24. Hagihara, T. et al. Assessment of Sentinel Node Concept in Esophageal Cancer Based on Lymph Node Micrometastasis. Ann Surg Oncol. 20, 3031-3037 (2013).

25. Jefferson, G. D., Sollaccio, D., Gomez-Fernandez, C. R. \& Civantos, F. Evaluation of immunohistochemical fine sectioning for sentinel lymph node biopsy in oral squamous cell carcinoma. Otolaryngol Head Neck Surg. 144, 216-9 (2011).

26. Lee, C. M. et al. Should lymph node micrometastasis be considered in node staging for gastric cancer? The significance of lymph node micrometastasis in gastric cancer. Ann Surg Oncol. 22, 765-71 (2015).

27. Kim, J. J. et al. Lymph node micrometastasis in node-negative early gastric cancer. Eur J Surg Oncol. 35, 409-14 (2009).

28. Li, Y. et al. Lymph node micrometastases is a poor prognostic factor for patients in pN0 gastric cancer: a meta-analysis of observational studies. J Surg Res. 191, 413-22 (2014).

29. Sonoda, H. \& Tani, T. Clinical significance of molecular diagnosis for gastric cancer lymph node micrometastasis. World J Gastroenterol. 20, 13728-33 (2014).

30. Huang, J. et al. The prognostic impact of occult lymph node metastasis in node-negative gastric cancer: a systematic review and meta-analysis. Ann Surg Oncol. 20, 3927-34 (2013).

31. Lee, C. M. et al. Should lymph node micrometastasis be considered in node staging for gastric cancer?: The significance of lymph node micrometastasis in gastric cancer. Ann Surg Oncol. 22, 765-71 (2015).

32. Blouhos, K. et al. Recurrence of a pT2N0cM0 Lower third gastric cancer with no. 6 lymph node micrometastasis after R0 extended surgery. Should adjuvant therapy be performed in conventionally node-negative but micrometastasis-positive pT2 gastric cancer? J Gastrointest Cancer. 48, 89-93 (2017).

33. Sasako, M. et al. Five-year outcomes of a randomized phase III trial comparing adjuvant chemotherapy with S-1 versus surgery alone in stage II or III gastric cancer. J Clin Oncol. 29, 4387-93 (2011).

34. Noh, S. H. et al. Adjuvant capecitabine plus oxaliplatin for gastric cancer after D2 gastrectomy (CLASSIC): 5-year follow-up of an open-label, randomised phase 3 trial. Lancet Oncol. 15, 1389-96 (2014).

\section{Author contributions}

F.A.V.S.: Substantial contributions to the conception and design of the work; the acquisition, analysis, and interpretation of data for the work; drafting the work; final approval of the version to be published; and agreement to be accountable for all aspects of the work in ensuring that questions related to the accuracy or integrity of any part of the work are appropriately investigated and resolved. A.P.D.L.: Substantial contributions to the design of the work; the analysis, and interpretation of data for the work; revising the work critically for important intellectual content; final approval of the version to be published; and agreement to be accountable for all aspects of the work in ensuring that questions related to the accuracy or integrity of any part of the work are appropriately investigated and resolved. A.J.A.W.: Substantial contributions to the design of the work; the analysis, and interpretation of data for the work; revising the work critically for important intellectual content; final approval of the version to be published; and agreement to be accountable for all aspects of the work in ensuring that questions related to the accuracy or integrity of any part of the work are appropriately investigated and resolved. M.A.D.F.: Acquisition, analysis, and interpretation of data for the work; drafting the work; final approval of the version to be published; and agreement to be accountable for all aspects of the work in ensuring that questions related to the accuracy or integrity of any part of the work are appropriately investigated and resolved. P.R.S.R.: Substantial contributions to the design of the work; the analysis, and interpretation of data for the work; revising the work critically for important intellectual content; final approval of the version to be published; and agreement to be accountable for all aspects of the work in ensuring that questions related to the accuracy or integrity of any part of the work are appropriately investigated and resolved. T.P.N.: Substantial contributions to the design of the work; the analysis, and interpretation of data for the work; revising the work critically for important intellectual content; final approval of the version to be published; and agreement to be accountable for all aspects of the work in ensuring that questions related to the accuracy or integrity of any part of the work are appropriately investigated and resolved.

\section{Competing interests}

The authors declare no competing interests.

\section{Additional information}

Correspondence and requests for materials should be addressed to F.A.V.S. 
Reprints and permissions information is available at www.nature.com/reprints.

Publisher's note Springer Nature remains neutral with regard to jurisdictional claims in published maps and institutional affiliations.

(c) (i) Open Access This article is licensed under a Creative Commons Attribution 4.0 International License, which permits use, sharing, adaptation, distribution and reproduction in any medium or format, as long as you give appropriate credit to the original author(s) and the source, provide a link to the Creative Commons license, and indicate if changes were made. The images or other third party material in this article are included in the article's Creative Commons license, unless indicated otherwise in a credit line to the material. If material is not included in the article's Creative Commons license and your intended use is not permitted by statutory regulation or exceeds the permitted use, you will need to obtain permission directly from the copyright holder. To view a copy of this license, visit http://creativecommons.org/licenses/by/4.0/.

(C) The Author(s) 2020 